\title{
ANALYSE SENSORIELLE COMPAREE DE DEUX PRODUITS DE FRITURES A L'HUILE VEGETALE ISSUS DE QUATRE NOUVEAUX HYBRIDES DE BANANE ET DE LA VARIETE ORISHELE
}

\author{
S. COULIBALY ${ }^{1}$, L.B. KOFFI' ${ }^{1}$, N. G. AMANI ${ }^{2}$ \\ ${ }^{1}$ Station de Recherche Technologique (SRT) du Centre National de Recherche Agronomique (CNRA), \\ 08 BP 881 Abidjan 08 Côte d'Ivoire. E-mail : coulisouley@yahoo.fr \\ 2 Unité de Formation et de Recherche / Sciences et Technologie des Aliments (UFR/STA). Université d'Abobo-Adjamé \\ 02 BP 801 ABIDJAN 02.
}

\begin{abstract}
RESUME
Les bananes hybrides CRBP 14, CRBP 39, FHIA 17 et FHIA 21, tolérantes à la cercosporiose et la variété Orishele ont fait l'objet d'évaluations sensorielles. Ces nouveaux hybrides et la variété de banane plantain Orishele du même degré de mûrissement, ont été comparés sous 2 formes de préparations culinaires. Les résultats des tests d'évaluation montrent que la banane hybride FHIA 17 n'est pas appréciée ni sous forme de chips ni sous forme d'Aloco (tranches molles frites à l'huile végétale). Par contre, les hybrides CRBP 14, CRBP 39 et FHIA 21, très appréciés sous ces 2 formes de préparations culinaires, peuvent valablement remplacer la variété Orishele, sensible à la cercosporiose.
\end{abstract}

Mots-clés : Evaluation sensorielle, hybride de banane, banane plantain, résistance à la cercosporiose.

\author{
ABSTRACT \\ SENSORIAL COMPARED ANALYSIS OF TWO WITH VEGETABLE OIL FRIED PRODUCTS FROM FOUR NEW \\ BANANA HYBRIDS AND ORISHELE VARIETY
}

Banana hybrids CRBP 14, CRBP 39, FHIA 17 and FHIA 21, resistant to cercosporiosis and the Orishele variety were tested for sensory evaluation. These new hybrids and the Orishele variety at the same maturity stage were cooked differently and compared. Sensory evaluation results show that hybrid FHIA 17 was not appreciated as chips nor as Aloco (soft slices fried in vegetable oil). But banana hybrids CRBP 14, CRBP 39, and FHIA 21 are well appreciated in these 2 culinary forms. So, they can replace the Orishele variety, wich was sensible to cercosporiosis.

Key words : Sensory evaluation, banana hybrid, plantain, resistance to cercosporiosis.

\section{INTRODUCTION}

Le genre Musa compte à ce jour 88 espèces et sous espèces, dont 46 cultivées et 42 sauvages, auxquelles il faut adjoindre 5 espèces connues mais non encore confirmées, ni définitivement classées (Daniels et al., 2001).

La plupart des espèces cultivées sont issues de Musa acuminata (génome A) et de Musa balbisiana (génome $B$ ). Suite à de nombreuses hybridations naturelles ou artificielles, des cultivars homo génomiques et allo génomiques, diploïdes, triploïdes et tétraploïdes ont vu le jour. Certains de ces cultivars sont sensibles à des maladies comme la cercosporiose noire, qui est préjudiciable à un bon rendement.

L'hybride CRBP 14 tétraploïde du groupe génomique $A A A B$ est à la fois tolérant aux charançons et partiellement résistant à la cercosporiose et à la fusariose. Le poids moyen de régime est $7,6 \mathrm{~kg}$. 
L'hybride CRBP 39 de type plantain tétraploïde $(A A A B)$ est obtenu par croisement entre un plantain femelle triploïde (Musa cv. AAB, variété locale «French clair») et un bananier mâle diploïde M23 hybride synthétique (AA). II présente un poids moyen de régime de $19,6 \mathrm{~kg}$ (Cohan et al., 2003). Il est à la fois tolérant aux charançons et partiellement résistant à la cercosporiose et à la fusariose.

L'hybride FHIA 17 du groupe génomique AAAA est quant à lui tolérant vis-à-vis du charançon du bananier (Cosmopolites sordidus Germar), résistant aux champignons Mycosphaerella fijiensis et Mycosphearella musicola (Molina et al., 2003). Par ailleurs, Gonzalez et al. (2003) ont montré sa résistance non seulement à la maladie des raies noires, mais aussi, à la maladie de Sigatoka (causée par M. musicola Leach) et à la fusariose (causée par Fusarium oxysporum $f$. sp cubense). FHIA 17 produit un régime dont le poids moyen est de $43,9 \mathrm{~kg}$ (Nowakunda et al., 2000 cités par Gonzalez et al., 2003).

L'hybride FHIA 21 du groupe génomique $A A A B$ est un bananier plantain résistant à la maladie des raies noires, à la fusariose et à la maladie de Sigatoka ; mais il est sensible aux nématodes. II possède un rendement élevé (22 à $27 \mathrm{~kg}$ par régime sans le rachis) et l'excellente qualité de ses fruits lui confère une place privilégiée, quant à la consommation en frais (au stade de maturité vert jaune) ou en cuit (au stade de maturité vert) (Guzmàn-Piedrahita et al., 2002).

Orishele est une variété cultivée du type Medium False horn, du groupe génomique $A A B$ et du sous-groupe plantain. Cette variété est régulièrement utilisée comme témoin pour des essais agronomiques par les chercheurs des 13 pays africains membres du réseau Musa pour l'Afrique Occidentale et Centrale (MUSACO) (Anonyme 2002). En outre, elle fait partie des 13 cultivars (sur les 1177 accessions existantes actuellement) stockés à long terme en toute sécurité en suspension cryoconservée, par le Consortium Mondial sur la Génomique de Musa (INIBAP, 2004).

Pour réduire l'incidence des maladies et des parasites sur le bananier plantain, des hybrides améliorés de banane ont été développés. Malheureusement ces nouveaux cultivars ne répondent pas toujours à l'attente des consommateurs, car les filières locales de transformation pour la consommation de la banane plantain sont d'une grande diversité (Foure et al., 1999). L'utilisation de la banane plantain à des fins culinaires diffère d'un cultivar à l'autre. Au Cameroun par exemple, certaines femmes peuvent en distinguer une dizaine. Selon l'utilisation prévue (recette, repas festif ou non) elles opèrent des choix complexes incluant des caractéristiques indispensables ou rédhibitoires (Dury et al., 2002). En Ouganda les variétés courantes de banane à cuire appelées «Matooke» constituent l'essentiel de la ration alimentaire des populations. Les variétés de banane améliorées ne sont pas cuisinées comme le «Matooke» car elles n'ont pas le même goût, selon les consommateurs (INIBAP 2003).

L'évaluation sensorielle de nouveaux hybrides et de nouveaux cultivars est l'une des étapes les plus décisives dans le processus de sélection et de création variétale. C'est une étape qui se situe entre la production des bananes en station et la diffusion des nouvelles plantules en milieu paysan. Elle permet de se prononcer sur l'acceptabilité des nouveaux produits par les futurs consommateurs.

L'objectif de notre étude est de déterminer l'acceptabilité par le consommateur ivoirien des hybrides CRBP 14, CRBP 39, FHIA 17 et FHIA 21 , qui sont à la fois tolérants aux charançons et partiellement résistants à la cercosporiose et à la fusariose (Nowakunda et al., 2000).

\section{MATERIEL ET METHODES}

\section{MATIERE PREMIERE}

Les tests d'évaluation ont porté sur les hybrides de banane CRBP 14, CRBP 39, FHIA 17 et FHIA 21, récoltés au stade de maturité maximale (Mitra, 1997), c'est à dire à l'apparition d'un doigt mûr sur le régime. Ces bananes ont le même degré de mûrissement selon l'échelle IPGRI INIBAP (IPGRI-INIBAP-CIRAD, 1996). La variété Orishele a été introduite dans les tests comme échantillon de référence caché (Watts et al., 1991).

\section{METHODES}

Les différentes préparations culinaires retenues sont l'Aloco ou banane frite et les chips, en raison de leur taux élevés de consommation ( $71 \%$ des personnes enquêtées consomment 
de l'Aloco comme goûter) au niveau de la population d'Abidjan (Amani et al., 2003). L'Aloco et les chips se préparent comme suit :

- Aloco ou banane plantain frite : Après lavage et épluchage, la pulpe de banane au stade de mûrissement «jaune» selon la charte de couleurs de IPGRI-INIBAP, est découpée en rondelles homogènes de $1 \mathrm{~cm}$ d'épaisseur. Ces rondelles sont ensuite frites pendant 5 minutes dans l'huile de palme décolorée, dont la température varie de 160 à $180{ }^{\circ} \mathrm{C}$. Les tranches d'Aloco ainsi obtenues sont molles.

- Chips : Pour la préparation des chips, l'on procède de la même manière que pour l'Aloco, mais avec de la banane au stade de mûrissement «vert moyen» (IPGRI-INIBAPCIRAD, 1996). Avec comme seules différences, des tranches plus minces (environ $0,3 \mathrm{~cm}$ d'épaisseur) et un temps de friture plus court (2 à 3 minutes). Les chips ainsi obtenus sont croustillants.

L'évaluation consiste en un test qui mesure l'intensité de différentes caractéristiques sensorielles. Ce test de notation mesure l'importance de la différence entre les échantillons et permet de classer ceux-ci par ordre croissant ou décroissant d'intensité d'une caractéristique donnée.

\section{Le panel de dégustateurs}

Le panel de dégustateurs est constitué d'une quinzaine de personnes des deux sexes toutes catégories sociales confondues, dont l'âge varie entre 25 et 45 ans. Tous les panélistes sont des consommateurs avertis des mets à évaluer. Ils n'ont reçu de formation que les explications liées à la bonne compréhension du test.

\section{Les questionnaires}

Plusieurs questionnaires ont été élaborés pour tenir compte des spécificités des mets à évaluer.

Les caractéristiques retenues pour les chips sont :

- Au niveau visuel : l'apparence et la couleur ;

- Avec les doigts : la friabilité et la fermeté ;

- Dans la bouche : la fermeté, la tendreté, l'effet craquant et le goût.
Les caractéristiques retenues pour l'Aloco sont :

- Au niveau visuel : l'apparence et la couleur ;

- Avec la fourchette : la fermeté et la plasticité ; - Avec les doigts : la tendreté, la friabilité et l'effet collant ;

- Dans la bouche : la tendreté, la fermeté, l'effet collant et le goût.

Les notes à attribuer sont comprises entre 1 et 5 et correspondent aux appréciations suivantes : $1=$ médiocre $; 2=$ mauvais $; 3=$ moyen ; $4=$ bon ; $5=$ très bon.

Toutes les caractéristiques doivent avoir le plus grand nombre de points pour indiquer la préférence d'une banane à une autre.

\section{Déroulement des tests d'évaluation}

Les échantillons codés avec des numéros aléatoires de 3 chiffres sont présentés dans un ordre choisi au hasard aux dégustateurs (Watts et al., 1991). Chaque échantillon a un numéro distinct.

II s'agit de tests de mesures d'intensité à 5 niveaux, où tous les échantillons sont présentés sur le même plateau aux dégustateurs, afin de leur permettre de réévaluer au besoin certains échantillons.

\section{Analyses statistiques}

Le test - $t$ - de Student a été utilisé pour la comparaison des moyennes. Après le calcul de la variance, le test de comparaison des moyennes a permis de savoir si les valeurs obtenues sont significativement différentes au seuil de 5 \% (Snedecor et al., 1984).

Ces analyses ont été faites à l'aide du logiciel de statistiques SPSS version 2.1.

\section{RESULTATS}

\section{CHIPS DES 5 CULTIVARS DE BANANE}

Les chips des 5 cultivars ont une belle apparence, les notes (de 2,87 à 3,56) étant très proches ou supérieures à la moyenne $(3,00)$ (figure 1). Seul l'aloco de I'hybride FHIA 17 avec 2,31 a une apparence inacceptable. 
La couleur des chips des hybrides CRBP 14, CRBP 39, FHIA 21 et de la variété Orishele ont des notes variant entre 3,06 pour les hybrides CRBP 14 et 39 , et 3,62 pour la variété Orishele. L'hybride FHIA 17 se distingue par la note basse de 2,00 .

Pour la friabilité entre les doigts, les chips des 5 cultivars de banane ont obtenu des notes se situant toutes autour de la moyenne 3,00 . Ces notes varient entre 2,94 pour les hybrides CRBP 14 et FHIA 21, et 3,25 pour l'hybride CRBP 39. Avec une note de 2,62 l'hybride FHIA 17 a une fragilité peu appréciée.

Au niveau de la fermeté entre les doigts, les notes attribuées par le panel de dégustateurs varient entre 2,75 et 3,37 respectivement pour I'hybride FHIA 21 et la variété Orishele.

Pour la fermeté dans la bouche, les chips des hybrides CRBP 14, CRBP 39, FHIA 21 et de la variété Orishele ont des notes variant entre 2,94 pour l'hybride FHIA 21 et 3,25 pour le CRBP 39. L'hybride FHIA 17 se distingue avec la note de 2,37 .

Les notes du panel de dégustateurs, pour la tendreté des chips dans la bouche des 5 cultivars de banane se situent entre 2,56 pour l'hybride CRBP 14 et 3,12 pour l'hybride FHIA 21 .

L'effet craquant des chips des 5 cultivars de banane a obtenu des notes inférieures à la moyenne $(3,00)$ pour les hybrides FHIA 17 et FHIA 21 (respectivement 2,56 et 2,50) et supérieures de la moyenne ( de 3,69 à 3,87 ) pour les autres cultivars.

Les chips des 5 cultivars ont un goût très appréciable, les notes de 3,31 à 3,81 étant supérieures à la moyenne 3,00 (figure 1). Seuls les chips de l'hybride FHIA 17 notés 2,17 ont un goût inacceptable.

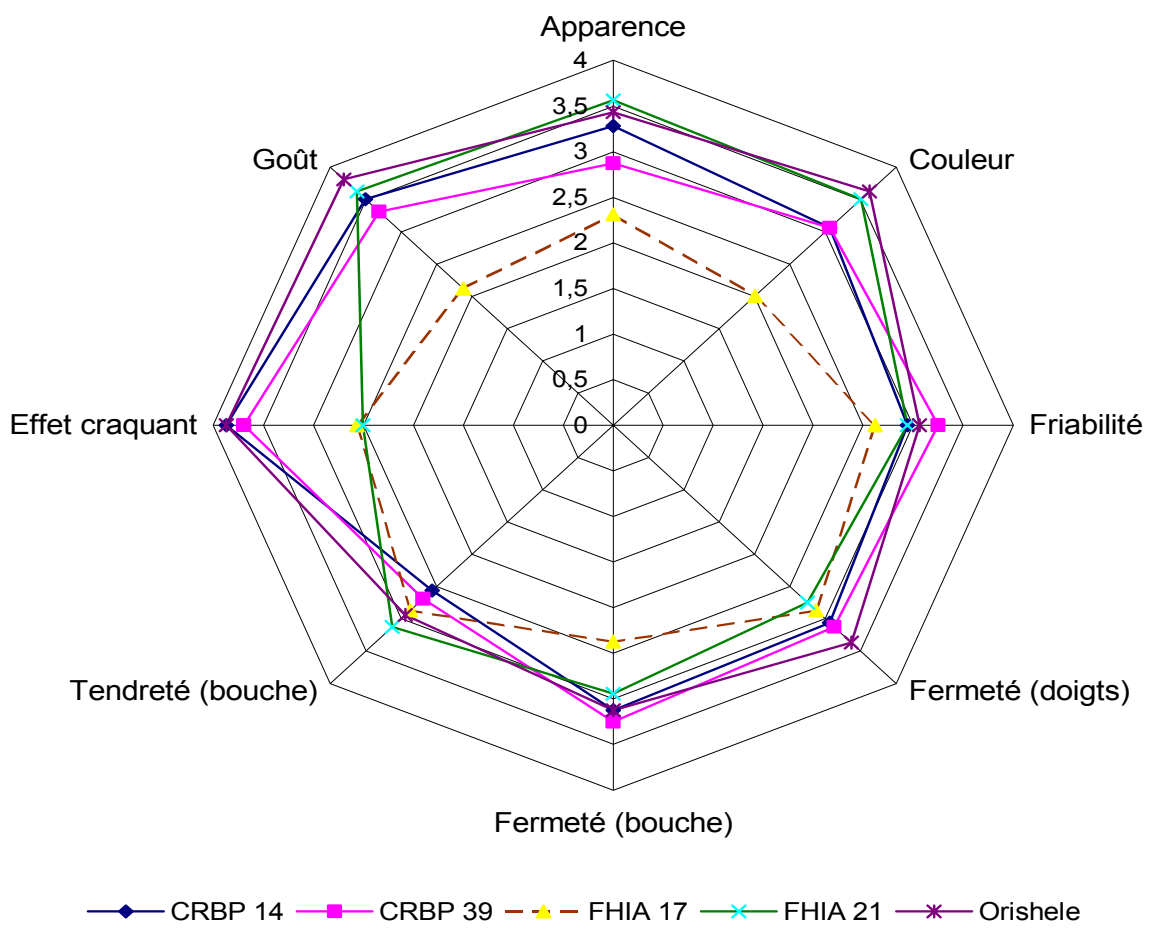

Figure 1 : Evaluation sensorielle des chips des 5 cultivars de bananes.

Sensorial evaluation of chips made from the 5 banana cultivars . 


\section{ALOCO DES 5 CULTIVARS DE BANANE}

Les aloco des 5 cultivars ont une belle apparence, les notes de 3,32 à 3,86 étant supérieures à la moyenne 3,00 (figure 2). Seule l'aloco de I'hybride FHIA 17 avec 2,07 a une apparence peu appréciée.

La couleur des aloco des hybrides CRBP 14, CRBP 39, FHIA 21 et de la variété Orishele ont des notes variant entre 3,36 pour le CRBP 39 et 3,93 pour le CRBP 14. L'hybride FHIA 17 se distingue par la plus faible note $(2,57)$.

Au niveau de la fermeté avec la fourchette, les notes attribuées par le panel de dégustateurs varient entre 3,36 et 2,93 respectivement pour les hybrides CRBP 14 et 39. L'hybride FHIA 17 avec une note de 2,50 , est encore moins apprécié pour cette caractéristique.

Le test de plasticité avec la fourchette donne pour les 5 cultivars des notes, qui se situent entre 2,57 pour les hybrides CRBP 14 et FHIA
17 et 3,00 pour les hybrides CRBP 39 et FHIA 21.

La tendreté au toucher des aloco des hybrides CRBP 14, FHIA 17 et FHIA 21 ont des notes variant entre 2,93 pour le $F H I A 17$ et 3,25 pour le FHIA 21. L'hybride CRBP 39 et la variété Orishele obtiennent respectivement 2,46 et 2,64 .

La friabilité entre les doigts des aloco des hybrides CRBP 39, FHIA 17 et de la variété Orishele ont des notes variant entre 2,04 pour la variété Orishele et 2,36 pour le CRBP 39 . Les hybrides CRBP 14 et FHIA 21 sont notés respectivement 2,71 et 3,00.

Au niveau de l'effet collant entre les doigts, les notes attribuées par le panel de dégustateurs sont de 3,00 pour l'hybride CRBP 14 et la variété Orishele, et de 3,32 pour l'hybride FHIA 21. Les hybrides CRBP 39 et FHIA 17 notés respectivement 2,54 et 2,39 sont peu appréciés.

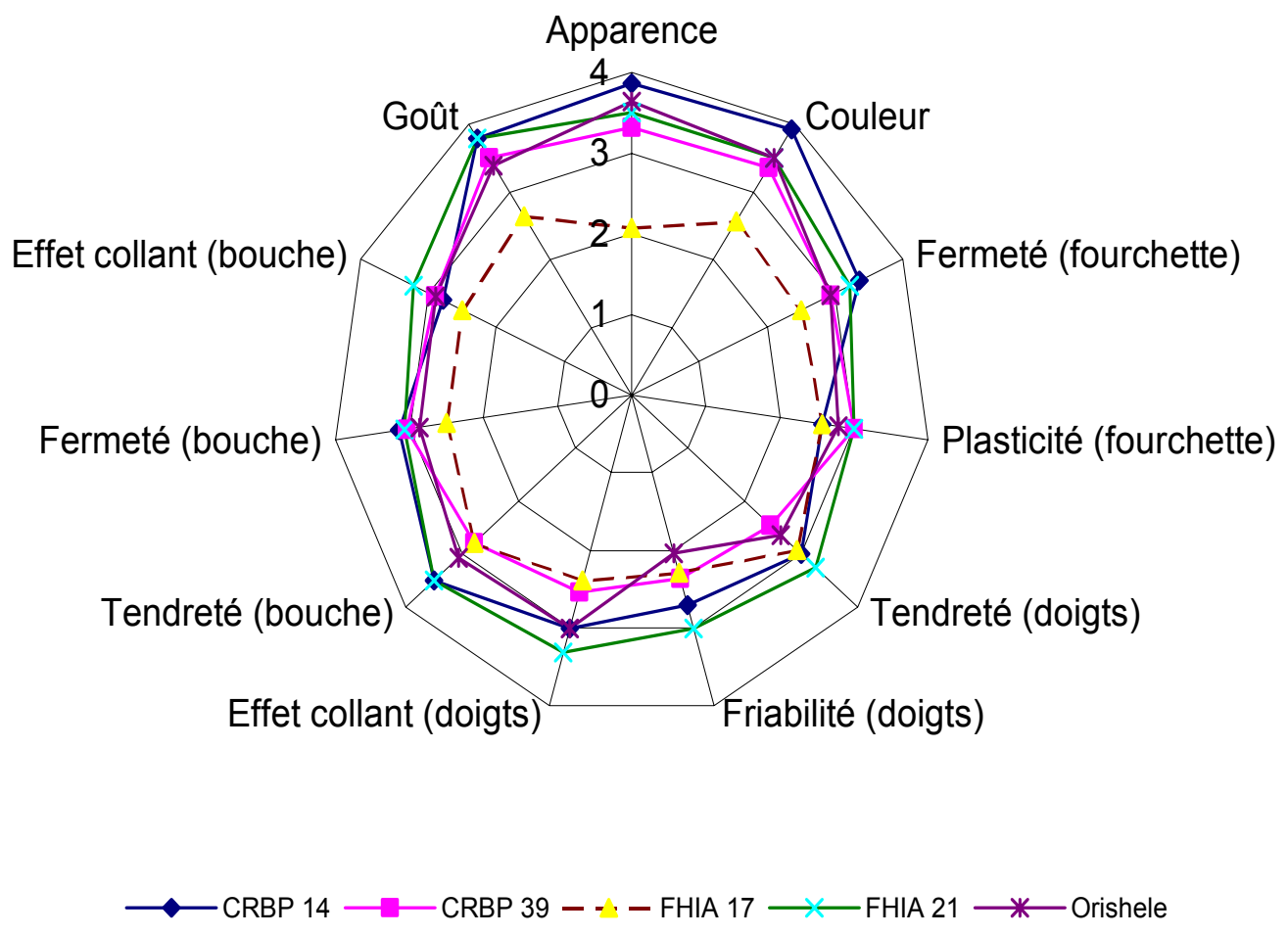

Figure 2 : Evaluation sensorielle de l'aloco des 5 cultivars de bananes.

Sensorial evaluation of aloco made from the 5 banana cultivars . 
La tendreté dans la bouche des aloco des hybrides CRBP 14 et FHIA 21 et de la variété Orishele ont des notes qui varient entre 3,07 pour la variété Orishele et 3,50 pour les hybrides CRBP 14 et FHIA 21. Les hybrides CRBP 39 et FHIA 17 obtiennent la note de 2,79.

Le test de fermeté dans la bouche donne pour les 5 cultivars des notes, qui se situent entre 2,50 pour l'hybride FHIA 17 et 3,14 pour l'hybride CRBP 14.

Pour l'effet collant dans la bouche, les aloco des hybrides CRBP 14, CRBP 39, FHIA 17 et de la variété Orishele ont des notes variant entre 2,50 pour l'hybride $F H I A 17$ et 2,89 pour la variété Orishele. L'hybride FHIA 21 est noté 3,21.

Le goût des aloco des hybrides CRBP 14, CRBP 39, FHIA 21 et de la variété Orishele ont des notes de 3,39 pour la variété Orishele et 3,79 pour le CRBP 14. L'hybride FHIA 17 se distingue par la plus faible note $(2,64)$.

Le tableau 1 indique les moyennes et les écarttypes des notes attribuées par les dégustateurs aux chips des différents cultivars de banane.

L'observation de ces résultats permet de faire 3 regroupements. Le premier groupe comprend les 2 hybrides CRBP 14 et 39. Dans le deuxième sont classés l'hybride FHIA 21 et la variété Orishele. L'hybride FHIA 17 constitue à lui seul le troisième groupe.
Le tableau 2 indique les moyennes et les écarttypes des notes attribuées par les dégustateurs aux aloco de différents cultivars de banane.

II est à remarquer que les aloco des hybrides CRBP 14, CRBP 39 et FHIA 21 ainsi que ceux de la variété Orishele, obtiennent au niveau de 6 des 11 caractéristiques évaluées, des notes dont les différences ne sont pas significatives au seuil de $5 \%$. II s'agit notamment de l'apparence, de la couleur, de la fermeté avec la fourchette, de la fermeté dans la bouche, de l'effet collant dans la bouche et du goût. Les notes pour toutes ces caractéristiques sont soit très proches, soit au dessus de la moyenne. Pour les mêmes caractéristiques, I'hybride FHIA 17 se singularise par des notes assez faibles dont les différences avec celles des autres cultivars sont significatives au seuil de $5 \%$.

Concernant les 5 autres caractéristiques (plasticité avec la fourchette, tendreté avec les doigts, friabilité avec les doigts, effet collant avec les doigts, et tendreté dans la bouche), la significativité des notes permet de toujours distinguer 2 groupes (désignés $A$ et $B$ ), où les différences ne sont pas significatives à l'intérieur du même groupe, mais elles le sont avec l'autre groupe. Ces 5 autres caractéristiques son présentées dans le tableau 3.

Tableau 1 : Moyennes et écart-types des notes (de 1 à 5) données par le panel de dégustateurs à des chips des 5 cultivars de banane.

Means and standard deviations of scores (from 1 to 5) given by the panel of tasters to the 5 banana cultivars chips.

\begin{tabular}{lccccc} 
& CRBP 14 & CRBP 39 & FHIA 17 & FHIA 21 & Orishele \\
\hline Apparence & $3,28 \pm 0,73 \mathrm{a}$ & $2,87 \pm 0,88 \mathrm{~b}$ & $2,31 \pm 1,08 \mathrm{~b}$ & $3,56 \pm 0,89 \mathrm{a}$ & $3,43 \pm 1,03 \mathrm{a}$ \\
Couleur & $3,06 \pm 0,77 \mathrm{a}$ & $3,06 \pm 0,93 \mathrm{a}$ & $2,00 \pm 0,40 \mathrm{~b}$ & $3,50 \pm 0,81 \mathrm{a}$ & $3,62 \pm 0,96 \mathrm{a}$ \\
Friabilité & $2,94 \pm 1,18 \mathrm{a}$ & $3,25 \pm 0,93 \mathrm{a}$ & $2,62 \pm 1,45 \mathrm{~b}$ & $2,94 \pm 0,93 \mathrm{a}$ & $3,06 \pm 1,18 \mathrm{a}$ \\
Fermeté (doigts) & $3,06 \pm 1,06 \mathrm{a}$ & $3,12 \pm 0,96 \mathrm{a}$ & $2,87 \pm 1,09 \mathrm{~b}$ & $2,75 \pm 0,86 \mathrm{~b}$ & $3,37 \pm 0,96 \mathrm{a}$ \\
Fermeté (bouche) & $3,12 \pm 0,96 \mathrm{a}$ & $3,25 \pm 0,93 \mathrm{a}$ & $2,37 \pm 0,96 \mathrm{~b}$ & $2,94 \pm 0,68 \mathrm{a}$ & $3,12 \pm 1,02 \mathrm{a}$ \\
Tendreté (bouche) & $2,56 \pm 0,81 \mathrm{a}$ & $2,69 \pm 0,95 \mathrm{a}$ & $2,87 \pm 1,15 \mathrm{a}$ & $3,12 \pm 0,89 \mathrm{~b}$ & $2,94 \pm 1,29 \mathrm{~b}$ \\
Effet craquant & $3,87 \pm 0,88 \mathrm{a}$ & $3,69 \pm 1,14 \mathrm{a}$ & $2,56 \pm 0,81 \mathrm{~b}$ & $2,50 \pm 0,82 \mathrm{~b}$ & $3,87 \pm 0,89 \mathrm{a}$ \\
Goût & $3,5 \pm 0,73 \mathrm{a}$ & $3,31 \pm 0,60 \mathrm{a}$ & $2,12 \pm 0,88 \mathrm{~b}$ & $3,62 \pm 0,96 \mathrm{a}$ & $3,81 \pm 0,75 \mathrm{a}$ \\
TOTAL & $25,39 \pm 7,12$ & $25,24 \pm 7,32$ & $19,72 \pm 7,82$ & $24,93 \pm 6,84$ & $27,22 \pm 8,08$ \\
\hline
\end{tabular}

Les valeurs d'une même ligne portant la même lettre ne sont pas significativement différentes au seuil de $5 \%$.

Values in the same liner with the same letter are not significantly different at $5 \%$ 
Tableau 2 : Moyenne et écart-type des notes (de 1 à 5) données par le panel de dégustateurs à l'aloco des 5 cultivars de banane.

Means and standard deviations of scores (from 1 to 5) given by the panel of tasters to aloco the 5 banana cultivars.

\begin{tabular}{|c|c|c|c|c|c|}
\hline & CRBP 14 & CRBP 39 & FHIA 17 & FHIA 21 & Orishele \\
\hline Apparence & $3,86 \pm 0,53 a$ & $3,32 \pm 1,03 a$ & $2,39 \pm 1,15 b$ & $3,5 \pm 0,94 a$ & $3,64 \pm 0,87 a$ \\
\hline Couleur & $3,93 \pm 0,47 a$ & $3,36 \pm 0,93 a$ & $2,79 \pm 0,70 b$ & $3,5 \pm 0,97 a$ & $3,50 \pm 1,04 a$ \\
\hline $\begin{array}{l}\text { Fermeté } \\
\text { (fourchette) }\end{array}$ & $3,36 \pm 0,74 a$ & $2,93 \pm 1,00 a$ & $2,50 \pm 0,76 b$ & $3,21 \pm 0,89 a$ & $2,93 \pm 0,47 a$ \\
\hline $\begin{array}{l}\text { Plasticité } \\
\text { (fourchette) }\end{array}$ & $2,57 \pm 0,65 a$ & $3,00 \pm 0,68 b$ & $2,57 \pm 0,76 a$ & $3,00 \pm 1,04 b$ & $2,79 \pm 0,73 a$ \\
\hline $\begin{array}{l}\text { Tendreté } \\
\text { (doigts) }\end{array}$ & $3,00 \pm 0,68 a$ & $2,46 \pm 0,63 b$ & $2,93 \pm 0,47 a$ & $3,25 \pm 0,94 a$ & $2,64 \pm 0,84 b$ \\
\hline $\begin{array}{l}\text { Friabilité } \\
\text { (doigts) }\end{array}$ & $2,71 \pm 0,91 a$ & $2,36 \pm 0,63 b$ & $2,29 \pm 0,83 b$ & $3,00 \pm 0,88 a$ & $2,04 \pm 1,05 b$ \\
\hline $\begin{array}{l}\text { Effet collant } \\
\text { (doigts) }\end{array}$ & $3,00 \pm 0,88 a$ & $2,54 \pm 0,84 b$ & $2,39 \pm 1,15 b$ & $3,32 \pm 0,87 a$ & $3,00 \pm 0,65 a$ \\
\hline $\begin{array}{l}\text { Tendreté } \\
\text { (bouche) }\end{array}$ & $3,50 \pm 0,76 a$ & $2,79 \pm 0,70 b$ & $2,79 \pm 0,70 b$ & $3,5 \pm 0,94 b$ & $3,07 \pm 0,85 b$ \\
\hline $\begin{array}{l}\text { Fermeté } \\
\text { (bouche) }\end{array}$ & $3,14 \pm 0,77 a$ & $3,04 \pm 0,80 a$ & $2,50 \pm 0,76 b$ & $3,07 \pm 1,00 \mathrm{a}$ & $2,86 \pm 0,86 a$ \\
\hline $\begin{array}{l}\text { Effet collant } \\
\text { (bouche) }\end{array}$ & $2,79 \pm 0,80 a$ & $2,90 \pm 0,77 a$ & $2,50 \pm 1,02 b$ & $3,21 \pm 0,97 a$ & $2,89 \pm 0,81 a$ \\
\hline Goût & $3,79 \pm 0,70 a$ & $3,50 \pm 0,76 a$ & $2,64 \pm 1,08 b$ & $3,79 \pm 0,70 a$ & $3,39 \pm 0,68 a$ \\
\hline TOTAL & $35,65 \pm 7,89$ & $32,2 \pm 8,77$ & $27,75 \pm 9,21$ & $36,35 \pm 10,41$ & $32,75 \pm 8,85$ \\
\hline
\end{tabular}

Les valeurs d'une même ligne portant la même lettre ne sont pas significativement différentes au seuil de $5 \%$.

Values in the same line with the same letter are not significantly different at $5 \%$.

Tableau 3 : Répartition des 5 cultivars de banane en fonction de la non significativité des différences des notes au niveau de certaines caractéristiques

Repartition of the 5 banana cultivars according to non signicance of score differences of some characteristics.

\begin{tabular}{cccccc}
\cline { 2 - 5 } & $\begin{array}{c}\text { Plasticité } \\
\text { (fourchette) }\end{array}$ & $\begin{array}{c}\text { Tendreté } \\
\text { (doigts) }\end{array}$ & $\begin{array}{c}\text { Friabilité } \\
\text { (doigts) }\end{array}$ & $\begin{array}{c}\text { Effet collant } \\
\text { (doigts) }\end{array}$ & $\begin{array}{c}\text { Tendreté } \\
\text { (bouche) }\end{array}$ \\
\hline \multirow{3}{*}{ Groupe A } & CRBP 14 & CRBP 14 & CRBP 14 & CRBP 14 & CRBP 14 \\
& FHIA 17 & FHIA 17 & FHIA 21 & $\begin{array}{c}\text { FHIA 21 } \\
\text { Orishele }\end{array}$ & FHIA 21 \\
\hline \multirow{3}{*}{ Groupe B } & FHIA 21 & CRBP 39 & CRBP 39 & CRBP 39 \\
& CRBP 39 & CRBP 39 & FHIA 17 & FHIA 17 & FHIA 17 \\
& FHIA 21 & Orishele & Orishele & & Orishele \\
\hline
\end{tabular}




\section{DISCUSSION}

Dans le premier groupe, au niveau les hybrides CRBP 14 et 39, les différences enregistrées ne sont pas significatives pour toutes les caractéristiques, exceptées celles de l'apparence des chips (tableau 1). Par ailleurs, toutes ces notes (de 2,87 à 3,87 ) sont très proches ou au dessus de la moyenne $(3,00)$, hormis celles de la tendreté $(2,56$ et 2,69$)$. Ces deux hybrides au stade de mûrissement «vert moyen» donnent des chips dont l'acceptabilité par le consommateur est donc sans équivoque. Ce résultat confirme celui de Cohan et al. (2003) qui citent l'hybride CRBP 39 parmi les bananes de type plantain.

Les cultivars du deuxième groupe (l'hybride FHIA 21 et la variété Orishele) présentent beaucoup de similitudes. En effet, toutes les moyennes (allant de 2,75 à 3,81 ) mis à part celle de l'effet craquant de l'hybride FHIA $21(2,50)$, sont proches.Par ailleurs il faut noter que pour la friabilité, la fermeté dans la bouche et le goût, les différences avec le premier groupe, ne sont pas significatives au seuil de $5 \%$. L'hybride FHIA 21 tout comme la variété Orishele sont donc bien indiqués pour la préparation des chips. $\mathrm{Ce}$ résultat est comparable à celui de Arcila (2002), qui indique une acceptabilité de $85 \%$ de l'hybride FHIA 21 sous diverses formes de préparations culinaires par le consommateur colombien.

L'hybride FHIA 17 qui constitue le troisième groupe (cf. tableau 1) se distingue par des notes généralement plus faibles (de 2,07 à 2,87 ) se situant en dessous de la moyenne $(3,00)$. Les différences avec les autres groupes sont généralement significatives au seuil de $5 \%$. L'hybride FHIA 17 donne donc des chips qui ne sont pas acceptés par le consommateur.

Pour les 5 caractéristiques citées au tableau 3 , les différences sont significatives entre les 2 hybrides CRBP 14 et CRBP 39. De même pour la tendreté, les différences sont toujours significatives pour les 2 hybrides FHIA 17 et FHIA 21. Les différences des notes ne sont pas par contre significatives pour les hybrides CRBP 14 et FHIA 21, mis à part la plasticité mesurée avec la fourchette.

II ressort par conséquent que contrairement aux 4 autres cultivars, l'acceptabilité de l'Aloco de l'hybride FHIA 17 par le consommateur n'est pas assurée. Ce résultat confirme le classement de Dens et al. (2002) de cet hybride parmi les bananes de type dessert.

Pour les chips, nos résultats sont comparables à ceux de Ssemwaga et al. (1995), et de Mwenebanda et al. (1996), cités par Nowakunda (2000), qui indiquent que les hybrides de banane ont de meilleures caractéristiques physiques que les cultivars locaux mais sont généralement moins appréciés pour leurs qualités culinaires.

\section{CONCLUSION}

Les caractéristiques étudiées permettent au vu des totaux des notes obtenues, tant au niveau des chips que des alocos des 5 cultivars de banane, de les classer par ordre décroissant de préférence des dégustateurs. Pour les chips on a le classement suivant :

- Orishele, CRBP 14, CRBP 39, FHIA 21 et FHIA 17.

Pour les alocos, le classement se présente comme suit :

\section{- FHIA 21, CRBP 14, Orishele, CRBP 39 et FHIA} 17.

II est à remarquer que pour ces 2 mets, l'hybride FHIA 17 est le moins apprécié. En outre, les totaux des notes obtenues $(19,72$ / 40 pour les chips et 27,75 / 55 pour les aloco) étant des valeurs très moyennes, cet hybride n'est pas indiqué pour la confection de ces 2 préparations culinaires. Les 4 autres cultivars par contre, aux totaux supérieurs aux moyennes $(25,24$ à 27,22 / 40 pour les chips et 32,20 à 36,35 / 55 pour les aloco), peuvent être utilisés pour la confection des ces 2 mets. La variété Orishele pourrait être ainsi valablement remplacée pour ce qui est de l'Aloco et des chips par les hybrides CRBP 14, CRBP 39 et FHIA 21, qui sont à la fois tolérants aux charançons et partiellement résistants à la cercosporiose et à la fusariose.

La poursuite de ces travaux permettra d'évaluer l'aptitude de ces nouveaux hybrides à la préparation d'autres mets locaux tels que le Foutou, le Foufou, le Concondé. Cette étude sera aussi judicieusement complétée par la détermination de leurs qualités nutritionnelles. 


\section{REFERENCES}

Amani G. et A. Kamenan. 2003. Potentialités nutritionnelles et technologie traditionnelle de tansformation des denrées amylacées en Côte d'Ivoire. In : IRD, FAO, Wageningen University (Eds.). Voies alimentaires d'amélioration des situations nutritionnelles en Afrique de l'Ouest: Le rôle des technologues alimentaires et des nutritionnistes. Deuxième Atelier International Ouagadougou, 23 - 28 novembre. Institut de recherche pour le développement, Wageningen University, FAO, $220 \mathrm{p}$

Anonyme. 2002. Cinquième réunion du comité de pilotage régional de Musaco. InfoMusa. $12(1): 61-62$.

Arcila Pulgarin M. I. 2002. Aceptabilidad por el consumidor de los platanos 'Africa 1' y 'FHIA-21' en el departamento del Quindío, Colombia. In : ACORBAT y COL (Eds.). Reunión de la associación para la cooperación en investigación de banano en el Caribe y en América tropical. Mem. $X V$ reunión. AUGURA, Asociación de Bananeros de Colombia, Medellín Reunión ACORBAT, Cartagena de Indias (COL), 10/ 27-11/02, AUGURA, Medellín Colombia, pp $578-581$.

Cohan J.-P., Abadie C., Tomekpe K. and J. Tchango-Tchango. 2003. Performances agrono-miques et résistance à la maladie des raies noires de l'hybride "CRBP 39». Infomusa. 12 (1) : 29 - 32.

Daniels J., Jenny C. and K. Karamara Tomekpe. 2001. Musalogue : a catalogue of Musa germplasm. in E. Arnaud and S. Sharrock (Eds.) Diversty in the genus Musa. Montpellier, France, International Network for the Improvement of Banana and Plantain, $213 p$.

Dens K., Vargas M., Matton G., Coessens S., Van de Houve I. et R. Swennen. 2002. Introduction et multiplication de bananiers et de bananiers plantain améliorés au Nicaragua et distribution aux agriculteurs. InfoMusa, 11 (1): 44 - 47.

Dury S., Bricas N., Tchango-Tchango J., Temple L. and A. Bikoi. 2002. The determinant of urban plantain consumption in Cameroon. Food Quality and Preferences. $13: 81-88$.

Foure E. et H. Tezenas Du Montcel. 1999. Les productions bananières - un enjeu économique majeur pour la sécurité alimentaire. Le Courrier $176: 71$ - 73 .

Gonzalez A. M. L., Gomez C. and L. Aristizábal 2003. Caractéristiques de croissance et de production d'hybrides FHIA en Colombie. InfoMusa, 12 (1) : 46 - 49.
Guzman-Piedrahita O. A. and J. Castaño-Zapata. 2002. Reconocimiento de nematodos fitopatógenos en plátanos 'Dominico hartón' (Musa AAB Simmonds), 'Africa', 'FHIA-20' y 'FHIA-21' en Colombia. Infomusa, 11 (2) : 33 - 36.

INIBAP. 2004. Rapport annuel. IPGRI, Rome Italy, INIBAP, Montpellier France, $44 \mathrm{p}$.

INIBAP. 2003. Rapport annuel. IPGRI, Rome Italy, INIBAP, Montpellier France, $40 \mathrm{p}$.

IPGRI, INIBAP and CIRAD. 1996. Descriptors for Banana (Musa spp.). International Plant genetic Resources Institute, Rome, Italy / International Network for the Improvement of Banana and Plantain, Montpellier, France / Centre de coopération internationale de recherche agronomique pour le développement, Montpellier, France, 55 p.

Khatijah I. and H. Patimah. 1999. Physico-chemical properties of native and cross-linked banana starches. Journal of Tropical Agriculture and Food Science, 27 (1) : 91 - 99.

Mokina T. O. I. and J. Castano-Zapata. 2003. Résistance des hybrides FHIA aux Mycosphaerella spp. Infomusa 12(2) : 25 28

Mwenebanda B. M. L. and D. Banda. 1996. The performance of advanced Musa yield trial at Limphasa in Nkhata-Bay District in Malawi. In Actes de la $15^{\text {th }}$ international conference on banana and plantain for Africa, (Kampala 14 - 18 octobre 1996), $10 \mathrm{p}$.

Mitra S. K. 1997. Postharvest physiology and storage of tropical and subtropical fruits. Wallingford, United Kindom : de CAB International, $423 \mathrm{p}$.

Nowakunda K., Rubaihayo P. R., Ameny M. A. et W . Tushemereiwe. 2000. L'acceptabilité des bananes exotiques par le consommateur ougandais. Infomusa : 9 (2) : 22 - 25.

Ssemwanga K. 1995. Quality attributes of matooke banana cultivars according to farmers and traders in Uganda. Musafrica 07 (7): 7 - 9.

Snedecor G. W. and W. G. Cochran. 1984. Méthodes statistiques. Trad. Boelle $\mathrm{H}$. and Camhaji E. $6^{\mathrm{e}}$ éd., Association de Coordination Technique Agricole, Imprimerie Laboureur et Cie Paris, 649 p.

Tomekpe K., Jenny C. And J.-V. Escalant. 2004. Revue des stratégies d'amélioration conventionnelle de Musa. Infomusa. $13(2): 2$ - 5.

Watts B. M., Ylimaki G. L., Jeffery L. E. and L. G. Elias. 1991. Méthodes de base pour l'évaluation sensorielle des aliments. CRDI. Ottawa, Canada, 145 p. 